\title{
Si beam-assisted graphitization of SiC (0001)
}

\author{
Piotr Ciochon ${ }^{1}$ [ · tukasz Bodek ${ }^{1} \cdot$ Mariusz Garb ${ }^{1} \cdot$ Łukasz Zając $^{1} \cdot$ Jacek J. Kołodziej ${ }^{1}$
}

Received: 22 June 2018 / Accepted: 28 September 2018 / Published online: 3 October 2018

(c) The Author(s) 2018

\begin{abstract}
We have performed a study of thermal graphitization of $\mathrm{SiC}$ (0001) surface in the high-purity molecular beam of Si atoms obtained from a controllable source. With the aid of Si beam, we have been able to achieve the semi-equilibrium reaction conditions and to control the growth rate of graphene film freely until complete stop of the graphitization, at the test temperature of $1350{ }^{\circ} \mathrm{C}$. Samples treated in Si beam are characterized by larger and homogeneous areas of graphene having uniform thickness, and much improved crystallographic ordering. This is attributed mainly to the improved buffer layer structure. The buffer layer shows neither disordered regions, nor point defects, which are prevalent in UHV-annealed samples. Significantly smaller concentrations of other surface defects are also observed. The used approach is a promising alternative to more commonly used buffer gas graphitization methods. Apart from the precise control for process parameters and very high process purity, it also allows for a co-deposition of other atoms at the growth stage.
\end{abstract}

\section{Introduction}

Graphitization of silicon carbide is one of the most widely used methods of the synthesis of graphene. It is particularly promising for electronic applications, because it yields the wafer-scale graphene readily on an insulating substrate, requiring no further transfer. As shown in the graphene roadmap [1], the graphitization technique is of particular interest for high-value applications, because of the large cost of the substrate and expensive processing (high processing temperature).

The process has been studied as early as 1961 [2] and is based on the high-temperature vacuum decomposition of the (0001) surface of $\mathrm{SiC}$ and the sublimation of more volatile silicon atoms [3]. Excess carbon atoms left on the surface undergo spontaneous transformation into a graphene film, separated from the substrate by a $(6 \sqrt{3} \times 6 \sqrt{3}) \mathrm{R} 30^{\circ}$ reconstructed buffer layer. The buffer layer contains atoms ordered similarly to the graphene lattice, but still covalently bound to the silicon atoms of the surface [4]. It is also responsible for the n-type doping of the synthesized graphene.

Piotr Ciochoń

piotr.ciochon@doctoral.uj.edu.pl

1 Faculty of Physics, Astronomy and Applied Computer Science, Institute of Physics, Jagiellonian University, ul. prof. Stanisława Łojasiewicza 11, 30-348 Kraków, Poland
Because the process is not self-limiting in UHV conditions, it has to be conducted at relatively low temperatures of around $1200{ }^{\circ} \mathrm{C}$ and during properly limited time, to avoid the formation of multi-layer graphene or graphite. However, in these temperatures, the mobility of carbon atoms on $\mathrm{SiC}$ surface is insufficient, resulting in the synthesis of the lowquality, inhomogeneous graphene film, having high concentration of defects, small area of monocrystalline domains and variable thickness of 1-6 ML $[5,6]$.

The most successful approach to overcome this limitation, so far, is conducting graphitization in the inert gases, e.g., argon, at atmospheric pressure which slows down the sublimation rate of Si atoms from the surface [7-9]. At these conditions, it is possible to use much higher process temperatures of around $1500{ }^{\circ} \mathrm{C}$, which result in higher mobility of carbon atoms and consequently in the higher-quality graphene layers, characterized by more uniform thickness of 1-2 ML, lower concentration of defects and large monocrystalline domains in the micrometer range.

In this paper, we propose an alternative approach to increase the graphitization process temperature, which relies on exposing $\mathrm{SiC}$ surface during the process to the molecular beam of silicon atoms from an external, high-purity $\mathrm{Si}$ sublimation source. This approach also reduces the effective sublimation rate of silicon from $\mathrm{SiC}$ surface, by resupplying $\mathrm{Si}$ atoms, but has several advantages over the buffer-gas method. 
Firstly, the use of even the purest available buffer gases at the atmospheric pressure results in the very large exposition of the silicon carbide surface to unknown impurities during graphitization (of the order of 1000 Langmuir/s, in case of the buffer gas of $6 \mathrm{~N}$ purity in atmospheric pressure). In contrast, the use of high-purity (MBE compatible) sublimation source (utilizing sublimation from the monocrystalline Si filament) results in the beam equivalent pressure (BEP) in the range of $10^{-7} \mathrm{mbar}$ and the BEP of the impurities in the range of $10^{-11}$ mbar in the worst-case scenario. This seems possibly a very significant improvement, especially in case of samples annealed for a prolonged period of time. Secondly, the areal density of the silicon atom flux can be adjusted freely, with high precision, and within a wide range of values, which creates the possibility to execute semi-equilibrium graphitization at high temperatures (for the context). This approach also allows for the co-deposition of other types of atoms on the graphitized surface, alongside the Si beam, which could lead to new strategies for doping and intercalating graphene on $\mathrm{SiC}$. The flux of $\mathrm{Si}$ atoms incoming to the surface can also be spatially varied, by tilting the sample or placing a patterned aperture in front of it, creating a potentially useful tool for different applications.

Unexpectedly, while the preparation of ordered $\mathrm{SiC}$ (0001) surfaces through annealing in the Si flux has been common and thoroughly described, we have not found papers reporting such attitude adopted for $\mathrm{SiC}$ graphitization. Somewhat similar ideas have been used only in course of few graphitization studies using disilane background gas $[10,11]$ and in the approach based on the controlled confinement of Si vapor near the surface of $\mathrm{SiC}$ during graphitization. In the latter method, Si confinement was achieved in several different ways, such as: conducting the process in a graphite enclosure with an aperture providing controllable leak of Si to the atmosphere [12], capping the SiC surface with another $\mathrm{SiC}$ substrate, which has been used to produce high-quality graphene on the C-face of the compound [13], or by capping the $\mathrm{SiC}$ with molybdenum plate during annealing, shown to induce heat accumulation at the surface, by reflecting thermal radiation, in addition to providing $\mathrm{Si}$ confimenent [14]. Other approaches to this process include: flash annealing in UHV [15] or in Pb atmosphere [16].

Here, we have performed a study of the Si beam-assisted $\mathrm{SiC}$ (0001) graphitization process, systematically varying its parameters, such as temperature, time and the Si flux density in search for a reliable technology capable of growing electronic-grade graphene films on a semiconducting substrate.

\section{Materials and methods}

Nominally undoped, semi-insulating, epi-ready $4 \mathrm{H} \mathrm{SiC}$ (0001) wafers obtained from University Wafer were used in the experiment. The samples were rinsed with isopropanol, blown dry with an inert gas and introduced into an ultrahigh vacuum (UHV) system (base pressure $<1 \times 10^{-10} \mathrm{mbar}$ ). They were degassed at the temperature up to $600{ }^{\circ} \mathrm{C}$ and subsequently annealed using an electron beam heater (acting from the backside of the $\mathrm{SiC}$ wafer) in the temperature range of $1100-1650{ }^{\circ} \mathrm{C}$ for $1-45 \mathrm{~min}$. The temperature was controlled using an infrared pyrometer, calibrated with the observation of melting point of silicon.

Most samples were annealed under silicon flux, with the control samples annealed in UHV conditions. An external sublimation source, SUSI 40 (MBE Komponenten), was used to produce the Si beam. The source is characterized by very high purity: all hot parts exposed to vacuum are made of silicon and the sublimation takes place from the monocrystalline filament (purity $>99.9999 \%$ ) The source is calibrated with the growth rate of silicon on a quartz crystal microbalance (QCM). This growth rate is then converted to Si flux density, using the known atomic density of Si [17]. The flux densities used in the experiments were within the range of $0.08-1.8 \times 10^{14} \mathrm{~cm}^{-2} \mathrm{~s}^{-1}$, or the BEPs (beam equivalent pressures) in the range of $10^{-8}-10^{-7} \mathrm{mbar}$ (derived from the QCM growth rate $1-20 \AA / \mathrm{min}$ ).

The process parameters (annealing temperature, time and silicon flux density), creating a three-dimensional parameter space, were varied systematically. The series of samples were characterized with a few complementary methods: low-energy electron diffraction (LEED), X-ray photoelectron spectroscopy (XPS), UV angle-resolved photoelectron spectroscopy (ARPES), and scanning tunneling microscopy (STM).

VG-SCIENTA R4000 hemispherical electron energy analyzer with a VUV5k UV lamp or a PREVAC RS 40B1 X-ray source using $\mathrm{Mg}$ anode source was used for photoelectron spectroscopy measurements, and OCI MCP LPS300-D diffractometer was used for LEED studies. The STM measurements were performed in a different UHV system (base pressure $<2 \times 10^{-10}$ mbar) equipped with an Omicron RT$\mathrm{STM} / \mathrm{AFM}$ microscope, using etched tungsten tips as probes. The samples were transferred between UHV systems in the medium vacuum suitcase $\left(p<1 \times 10^{-6}\right.$ mbar $)$.

Analysis of XPS data was performed using CasaXPS 2.3.16 [18], ARPES data using IgorPro 6.1.2.1 software [19] and STM images were processed using Gwyddion version 2.42 [20] and Fiji software [21].

\section{Results and discussion}

\subsection{Graphene growth kinetics}

To confirm the decrease in the growth rate of graphene under the flux of $\mathrm{Si}$ atoms and to assess the graphene growth 

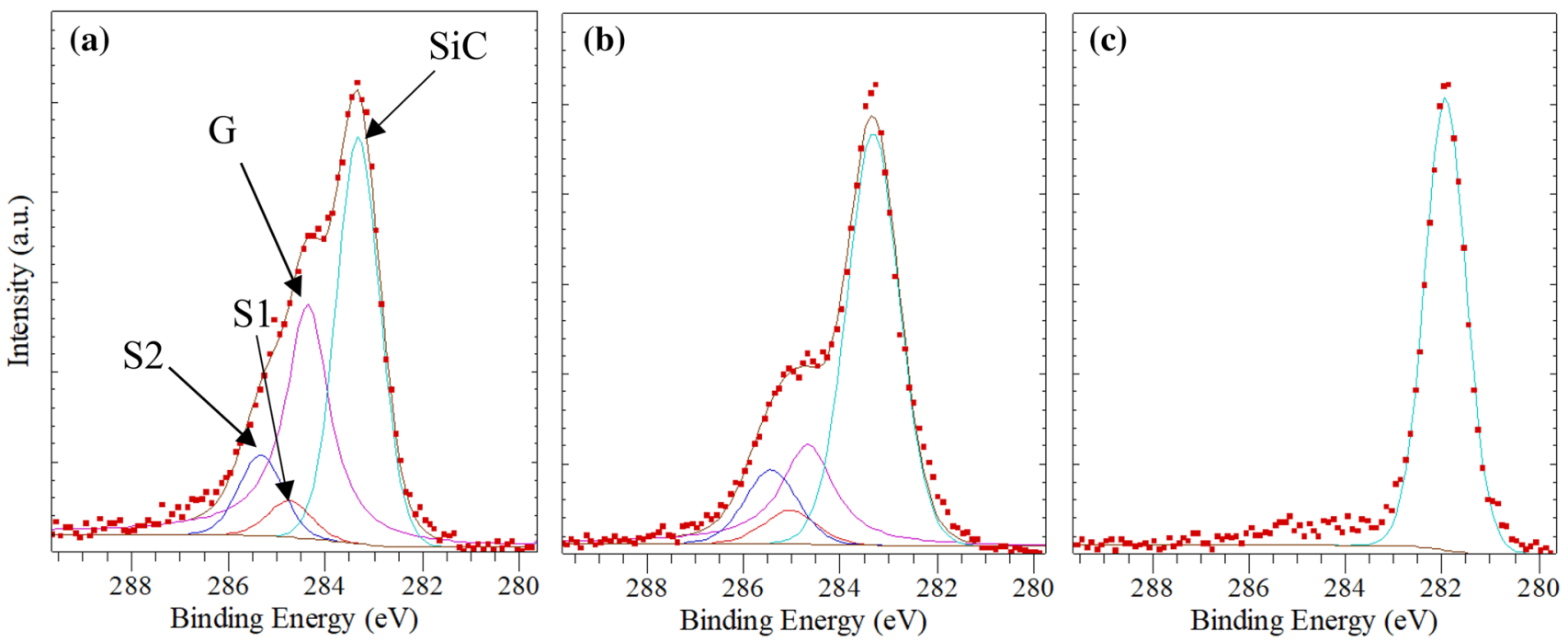

Fig. $1 \mathrm{C} 1 \mathrm{~s}$ region of an XPS spectrum of the sample of $\mathrm{SiC}(0001)$ annealed at $T=1350{ }^{\circ} \mathrm{C}$ for 5 min a in UHV, b in the Si flux of $1.0 \times 10^{14}$ $\mathrm{cm}^{2} \mathrm{~s}^{-1}, \mathbf{c}$ in the Si flux of $1.8 \times 10^{14} \mathrm{~cm}^{2} \mathrm{~s}^{-1}$

kinetics in different conditions, we used X-ray photoelectron spectroscopy.

Figure 1 shows the spectra of $\mathrm{SiC}$ (0001) samples annealed at the temperature of $1350{ }^{\circ} \mathrm{C}$ for $5 \mathrm{~min}$ in UHV (1a) and in the increasing flux of Si atoms (1b, 1c). The $\mathrm{C} 1 \mathrm{~s}$ peak consists of four main components, described in Fig. 1a, which can be associated with: bulk SiC (located at $E_{\mathrm{b}} \approx 283.4 \mathrm{eV}$ ), graphene (located at the binding energy: $E_{\mathrm{b}}$ $\approx 284.2 \mathrm{eV})$ and with the interface atoms: $\mathrm{S} 1\left(E_{\mathrm{b}} \approx 285 \mathrm{eV}\right)$ and $\mathrm{S} 2\left(E_{\mathrm{b}} \approx 285.6 \mathrm{eV}\right)[17,22]$. The graphene component is fitted using Doniach-Sunjic profile (numerically convoluted with a Gaussian function), because of its metallic behavior, while all the other peaks have a Gaussian-Lorentzian profile. The surface coverage of $\mathrm{SiC}$ with graphene is proportional to the intensity of the $\mathrm{G}$ component and can be used to quantitatively assess the growth rate of graphene.

As seen in Fig. 1, annealing in UHV resulted in the average coverage of one monolayer of graphene, while utilizing the moderate Si flux of $\Phi=1.0 \times 10^{14} \mathrm{~cm}^{2} \mathrm{~s}^{-1}$ considerably slowed down the graphitization-deconvolution of the spectrum showed the presence of interface components (associated with the buffer layer and the top $\mathrm{SiC}$ layer) and the $\mathrm{G}$ component, with its intensity reduced by around $45 \%$. The presence of the buffer layer and relatively small regions of graphene on the surface was also confirmed by LEED and ARPES studies. Applying larger Si flux of $1.8 \times 10^{14} \mathrm{~cm}^{-2} \mathrm{~s}^{-1}$ completely inhibited the graphitization and the $\mathrm{C} 1 \mathrm{~s}$ spectrum could be fitted with a single $\mathrm{SiC}$ peak. We also observed a significant shift of its position to the lower binding energies (by $1.4 \mathrm{eV}$ ), most likely due to band bending effects.
To study the growth kinetics, we have performed studies of the samples annealed at constant temperature, in varying incoming flux of $\mathrm{Si}$ atoms. Figure 2 shows the nominal atomic concentration (in this case equal to the percentage of the area of the whole peak) of the $\mathrm{G}$ component in the $\mathrm{C} 1 \mathrm{~s}$ spectrum of graphene, grown at the temperature of $1350{ }^{\circ} \mathrm{C}^{1}$ in: UHV (red) Si flux of $\Phi_{1}=1.0 \times 10^{14} \mathrm{~cm}^{-2} \mathrm{~s}^{-1}$ (green) and $\Phi_{2}=1.8 \times 10^{14} \mathrm{~cm}^{-2} \mathrm{~s}^{-1}$ (blue).

The chart of the sample annealed in the highest Si flux confirms that the graphitization is completely inhibited and the graphene does not appear on the surface even after prolonged annealing for $t=45 \mathrm{~min}$. The other two charts can be fitted rather accurately with the logistic-type function, according to the equation:

$y=\frac{A_{1}-A_{2}}{1+\left(x / x_{0}\right)^{p}}+A_{2}$.

This equation does not have immediate, physical meaning, but qualitatively well describes the observed phenomena. The growth kinetics appear to happen in two phases. In the beginning, the rate of reaction is inversely proportional to the amount of non-graphitized carbon present on the surface (kinetics resembling first-order, self-limiting chemical reaction) and its change over time is approximately proportional to $\frac{1}{1+\exp (-t)}$. However, the reaction is not completely self-limiting. When the coverage of the sample with

\footnotetext{
${ }^{1} 1350{ }^{\circ} \mathrm{C}$ was found to be the highest temperature, at which graphene growth could be completely inhibited with the maximal Si flux achievable from our source and it is of no meaning beside this.
} 


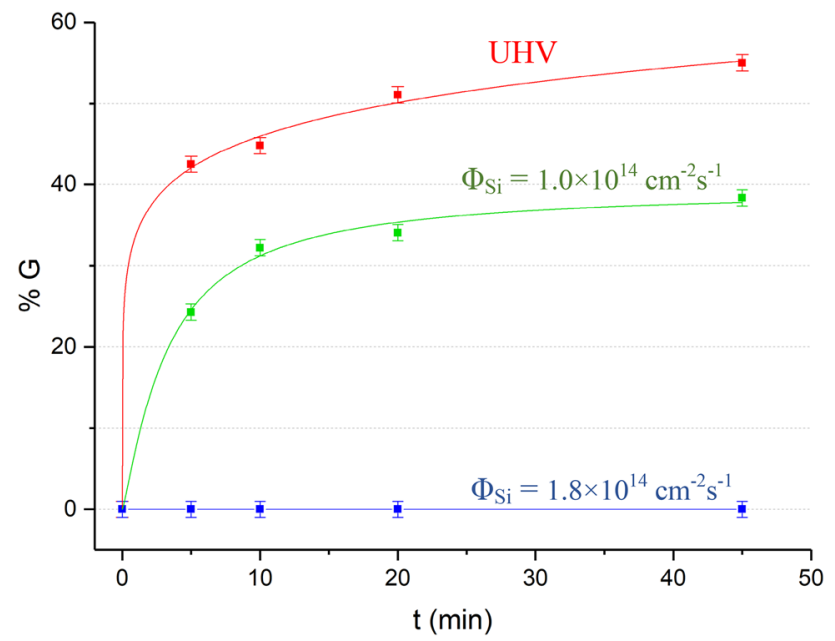

Fig. 2 Atomic concentration (\%) of the $\mathrm{G}$ component in the $\mathrm{C} 1 \mathrm{~s}$ spectrum of the $\mathrm{SiC}$ samples annealed at $T=1350{ }^{\circ} \mathrm{C}$ in UHV (red), in the Si flux of $\Phi_{1}=1.0 \times 10^{14} \mathrm{~cm}^{-2} \mathrm{~s}^{-1}$ (green) and $\Phi_{2}=1.8 \times 10^{14} \mathrm{~cm}^{-2} \mathrm{~s}^{-1}$ (blue)

graphene increases, the reaction progresses to the second phase, during which the reaction rate becomes approximately independent of the amount of non-graphitized carbon. It is instead limited by the ability of silicon atoms from the subsurface layers to diffuse through the defects present in the continuous graphene film, covering the surface. This leads to the approximately linear growth, with significantly decreased growth rate (resembling zero-order chemical reaction). This type of reaction kinetics, for both UHV and moderate $\mathrm{Si}$-flux conditions is consistent with the current understanding of the growth of epitaxial graphene on $\mathrm{SiC}(0001)$. Applying flux of $\mathrm{Si}$ atoms during graphitization decreases both the initial reaction rate (in the fast-growth phase) and the linear slope describing graphene growth in the slowgrowth phase.

These results show that $\mathrm{Si}$ beam-assisted graphitization allows us to freely and precisely adjust the growth rate of graphene. This opens a possibility of semi-equilibrium graphitization (in the case discussed above, at $T=1350{ }^{\circ} \mathrm{C}$, it took around $75 \mathrm{~min}$ to grow a single monolayer of graphene when it was graphitized in Si flux, compared to $5 \mathrm{~min}$ in UHV conditions; the resulting approximate growth rate is $0.015 \mathrm{ML} / \mathrm{min}$ ) and studying phenomena related to this very slow growth. Semi-equilibrium graphitization can also be extremely useful to study the atomistic mechanism of graphene growth, which is still not fully understood, as well as the kinetics of this inherently non-equilibrium process. We emphasize here, that, in such conditions, using the flux of $\mathrm{Si}$ atoms has two significant advantages over other methods: (1) exposition of the surface to the impurities is minimal, (which is particularly important during long exposition times, as impurities may affect the observed reactions at the atomic

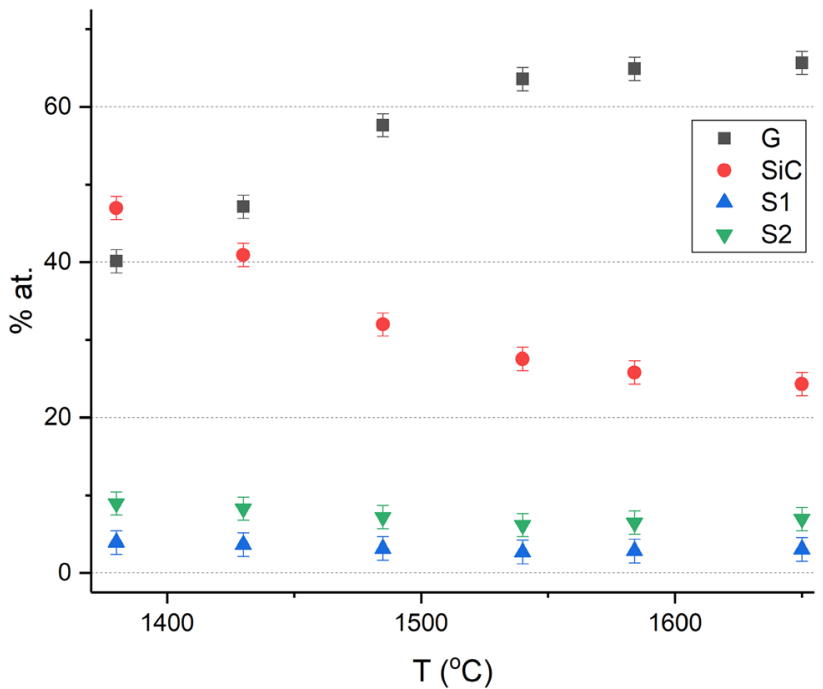

Fig. 3 Atomic concentration (\%) of the different components of $\mathrm{C} 1 \mathrm{~s}$ peak of $\mathrm{SiC}$ samples annealed for a constant time $(5 \mathrm{~min})$ and with constant Si flux of $1.8 \times 10^{14} \mathrm{~cm}^{-2} \mathrm{~s}^{-1}$, at varied temperature

scale) and (2) it allows for a very precise control over the effective reaction rate.

Graphene growth can be also performed in the "far from the equilibrium" conditions, with the assistance of the flux of $\mathrm{Si}$ atoms. This might be motivated by practical reasons (shorter annealing time needed to grow graphene samples) or by the intention to avoid some of the obstacles associated with prolonged annealing, such as step bunching. To investigate these non-equilibrium processes, we have prepared a series of samples annealed in systematically varied conditions (temperature, time, incoming Si flux). The results, showing the nominal atomic concentration of the different components of $\mathrm{C} 1 \mathrm{~s}$ spectrum for such series of samples, prepared in constant process time $(5 \mathrm{~min})$ and constant Si flux of $\Phi=1.8 \times 10^{14} \mathrm{~cm}^{-2} \mathrm{~s}^{-1}$, but at varied temperature (in the range of $1385-1650{ }^{\circ} \mathrm{C}$ ), are shown in Fig. 3.

Graphene coverage, proportional to the XPS nominal atomic concentration of the $\mathrm{G}$ component, increases with increasing temperature and stabilizes in the given conditions (time, Si flux) at temperatures exceeding $1600{ }^{\circ} \mathrm{C}$, at the nominal concentration of around $65 \%$. The nominal concentration of S1 and S2 components exhibits a slight decreasing trend with increasing annealing temperature, which is expected, given that the signal from the interface layer is attenuated by the increasing thickness of graphene. However, the magnitude of this effect is too small for accurate observation.

Coverage of the surface corresponding to a complete mono- and bilayer graphene, at the $G$ values of $42.5 \%$ and $62.5 \%$ respectively, was found using angle-resolved photoelectron spectroscopy (See Fig. 4a. for the relevant ARPES 


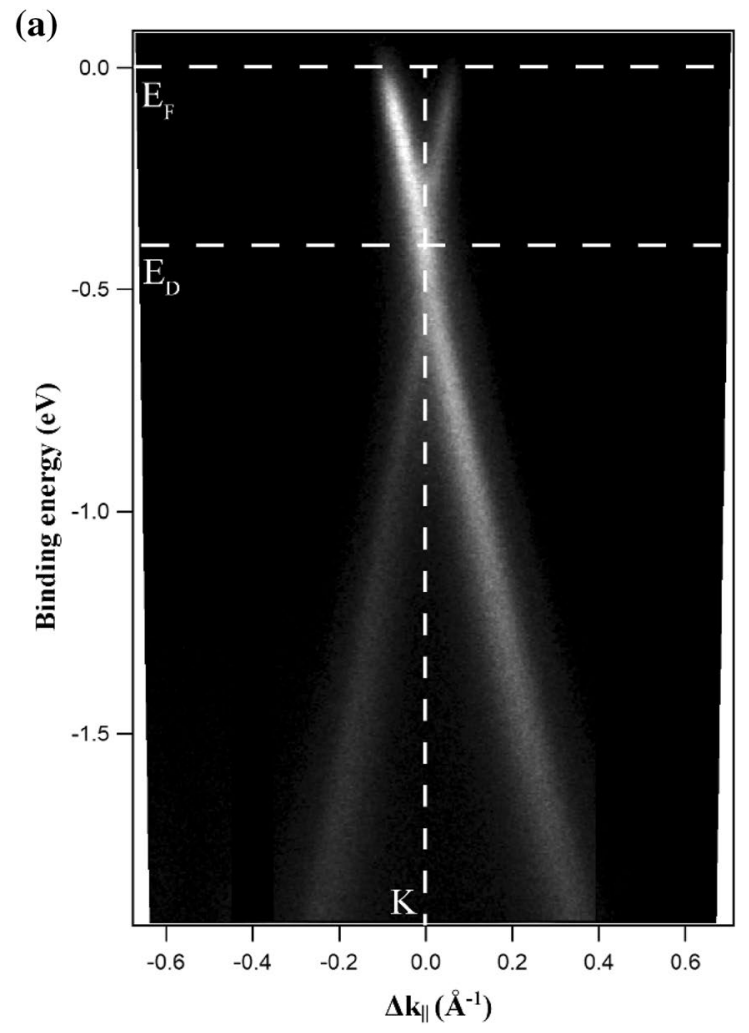

Fig. 4 ARPES spectra around the $\mathrm{K}$ point in the reciprocal lattice of graphene for samples annealed for $5 \mathrm{~min}$, in the Si flux equal to $1.8 \times 10^{14} \mathrm{~cm}^{-2} \mathrm{~s}^{-1}$ a at $1430{ }^{\circ} \mathrm{C}$, showing a single dispersion relation characteristic for the monolayer graphene, and $\mathbf{b}$ at $1580{ }^{\circ} \mathrm{C}$, showing

spectra). ARPES measurements have also shown that the graphene is n-doped, with Dirac point located around $0.4 \mathrm{eV}$ below the Fermi level, which is consistent with the graphene prepared on silicon carbide with other methods [23], and shows that there is no intercalation of graphene with silicon atoms (the process of the intercalation of the graphene grown on $\mathrm{SiC}$ (0001) with $\mathrm{Si}$ is reported in Ref. [24]).

\subsection{Surface structure-STM studies}

Figure 5a, b shows the STM images of two different areas on the SiC sample annealed at $T=1400{ }^{\circ} \mathrm{C}$ for $5 \mathrm{~min}$ in UHV conditions, while Fig. $5 \mathrm{c}, \mathrm{d}$ show images of $\mathrm{SiC}$ annealed in the same conditions, but with an Si flux of $\Phi=0.4 \times 10^{14} \mathrm{~cm}^{-2} \mathrm{~s}^{-1}$. The areas of monolayer graphene are marked as ML, bilayer graphene as BL, and amorphous areas as A. The assignment is based on the observed step heights and characteristic STM patterns.

Earlier STM studies reported that the apparent STM step height between the monolayer and bilayer graphene was $0.6-0.9 \AA[25,26]$. This is much lesser than the interlayer spacing observed for the graphite $(3.35 \AA)$ or $\mathrm{SiC}$ bilayer height $(2.5 \AA)$ and, therefore, reliably identifies the

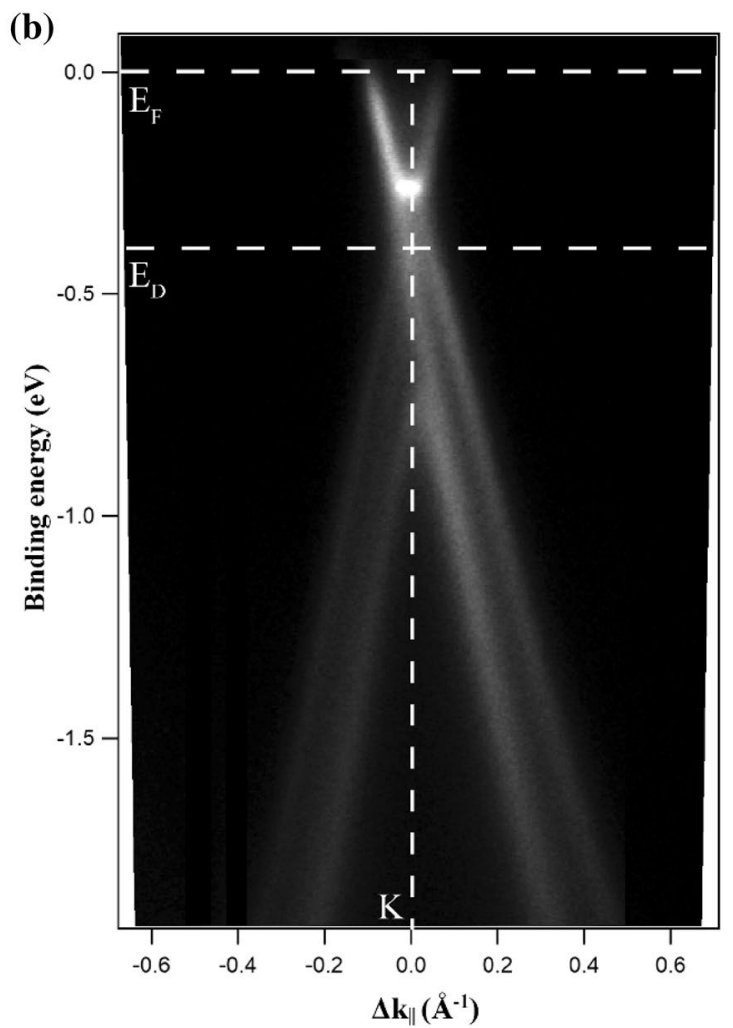

a two-band dispersion relation characteristic for the bilayer graphene; Fermi energy $\left(E_{\mathrm{F}}\right)$, Dirac energy $\left(E_{\mathrm{D}}\right)$ and $K$ point in the reciprocal lattice are marked in the figure

coverages under discussion. This small apparent step height has been attributed to electronic effects interfering with the topography during STM imaging [24] or to the bottom-up growth of epitaxial graphene [25]. According to these lines, the monolayer and bilayer graphene areas are identified as those which are separated with the STM step having a height of around $0.8 \AA$ (see an example of a line profile across the bi/monolayer boundary, shown in the inset in Fig. 5a).

The amorphous areas, observed for the sample annealed in UHV, have the form of pits, with apparent (STM) depth of around $7.8 \AA$, equal to around three bilayers of $\mathrm{SiC}$ (7.5 $\AA$ ). We also observed few amorphous pits for the surfaces annealed in Si flux, but they were usually shallower, with the depth of around 5-6 $\AA$ (around 2 bilayers of $\mathrm{SiC}$ ). Surface pits have been observed for the graphene/SiC $(0001)$ system, but they were usually somewhat deeper [23]. The double step observed in Fig. 5c) has an apparent height of around $10 \AA$ (each step has a height of around $5 \AA$ ), while the step observed in Fig. $5 \mathrm{~d}$ has a height of around $5 \AA$. These can be attributed to steps with a height of two SiC bilayers (2.5 ̊) [27].

Overall, the morphology of the samples appears to be significantly improved by applying the flux of $\mathrm{Si}$ atoms during 

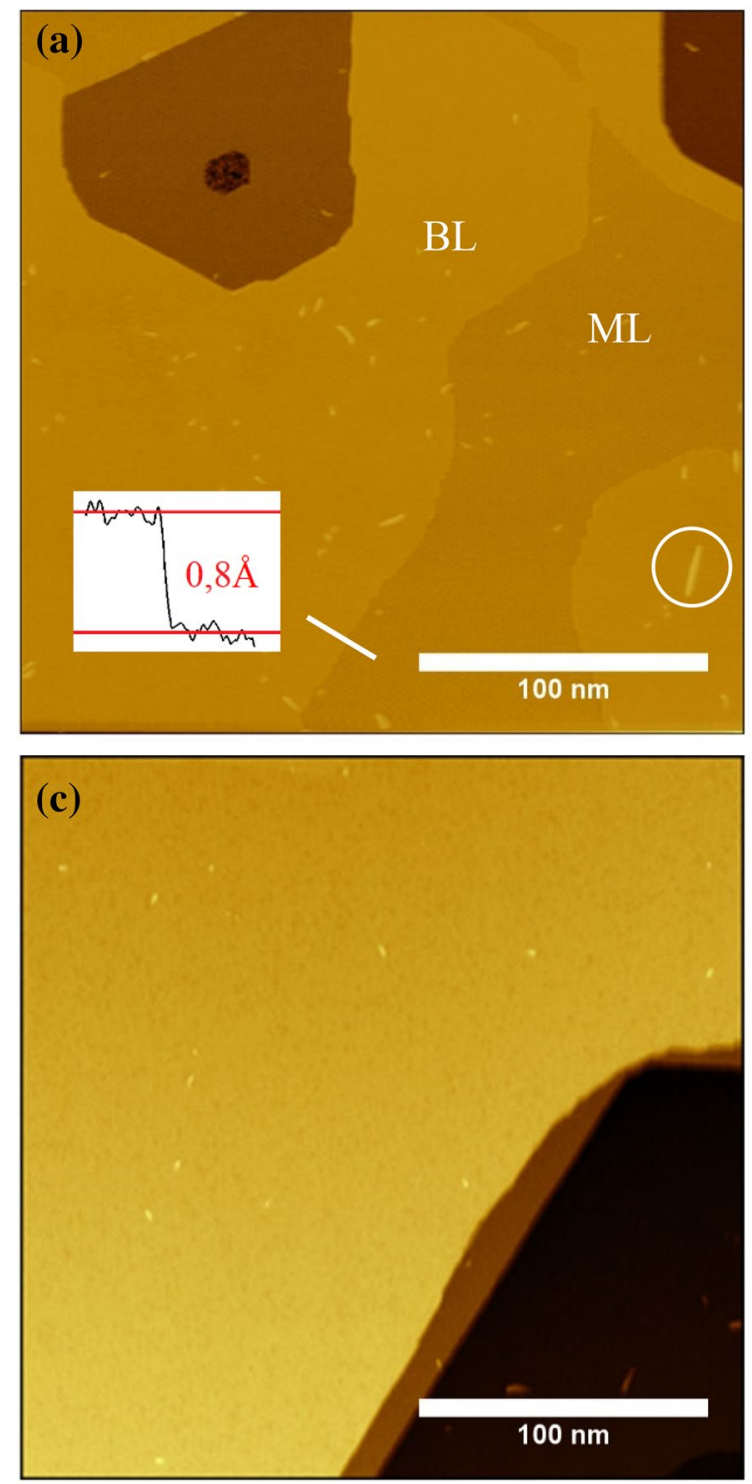

Fig. 5 STM images of $\mathrm{SiC}$ samples annealed in $T=1400{ }^{\circ} \mathrm{C}$ for 5 min: a, b without $\mathrm{Si}$ flux, c, $\mathbf{d}$ in $\mathrm{Si}$ flux of $0.4 \times 10^{14} \mathrm{~cm}^{-2} \mathrm{~s}^{-1}$. Imaging parameters are: a $I=12 \mathrm{pA}, U=400 \mathrm{mV}$; b $I=15 \mathrm{pA}$,

graphitization. The samples annealed in a moderately low Si flux of $\Phi=0.4 \times 10^{14} \mathrm{~cm}^{-2} \mathrm{~s}^{-1}$ (far from the semi-equilibrium conditions at this temperature) exhibit a larger degree of surface order and larger areas of continuous, monolayer graphene. We observed some amorphous pits on the surface, but their number was reduced compared to UHV conditions and they were usually shallower. Interestingly, the amorphous pits appear to be positioned near the step edges for the Si beam-assisted growth, while their distribution for the UHV-grown samples is rather uniform across the surface.

We also observed a large reduction of the amount and size of point defects present on the surface (example marked by circle in Fig. 5a). These defects are commonly observed for

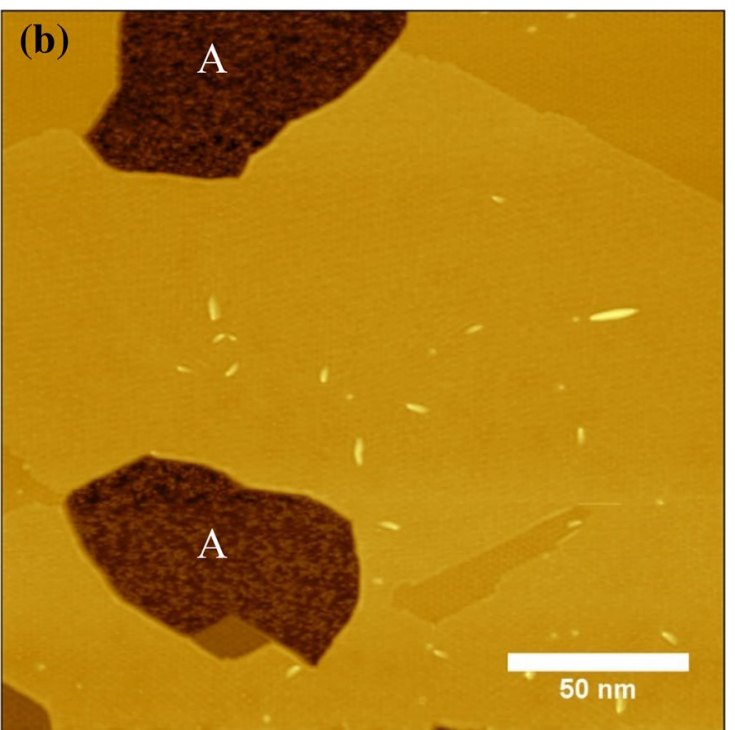

(d)

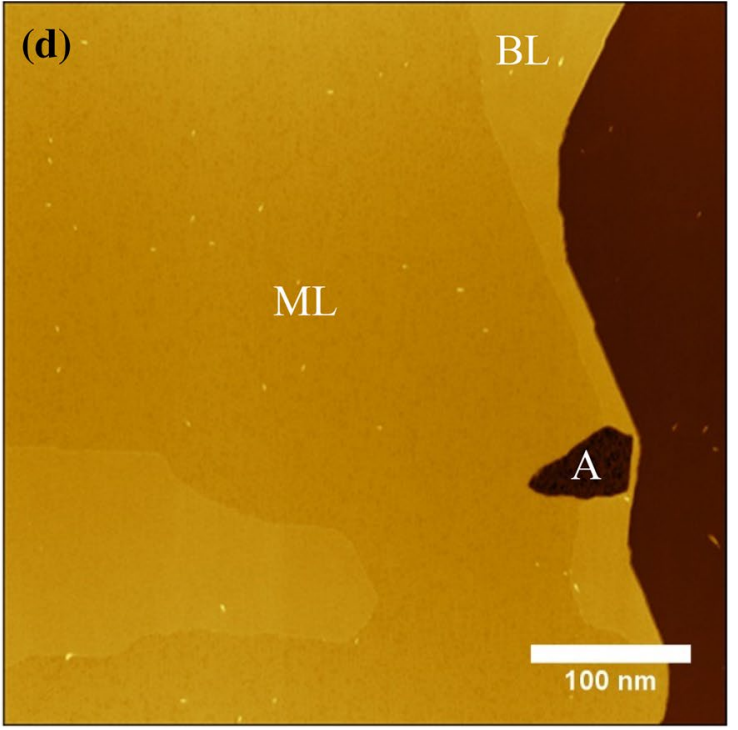

$U=400 \mathrm{mV} ; \mathbf{c}, \mathbf{d} I=20 \mathrm{pA}, U=400 \mathrm{mV}$; inlet in Fig. 5a shows the height profile along the white line marked in the figure

the graphene/SiC (0001) system and they were attributed either to carbon nanotube-type defects [28] or to the other off-plane defects in epitaxial graphene. Regardless of their origin, it is evidenced that Si flux-assisted growth is effective in reducing their density.

To gain more insight into the properties of graphene grown in different conditions, we performed atomically resolved STM studies: Fig. 6a, b shows the SiC sample annealed in the flux of $\mathrm{Si}$ atoms, while Fig. 6c, d shows the sample annealed in vacuum. An atomically resolved graphene structure is visible as a fine grid with a lattice constant of around $0.25 \mathrm{~nm}$, overlaid on the grid with a much larger lattice constant of around $3.1 \mathrm{~nm}$, consistent 

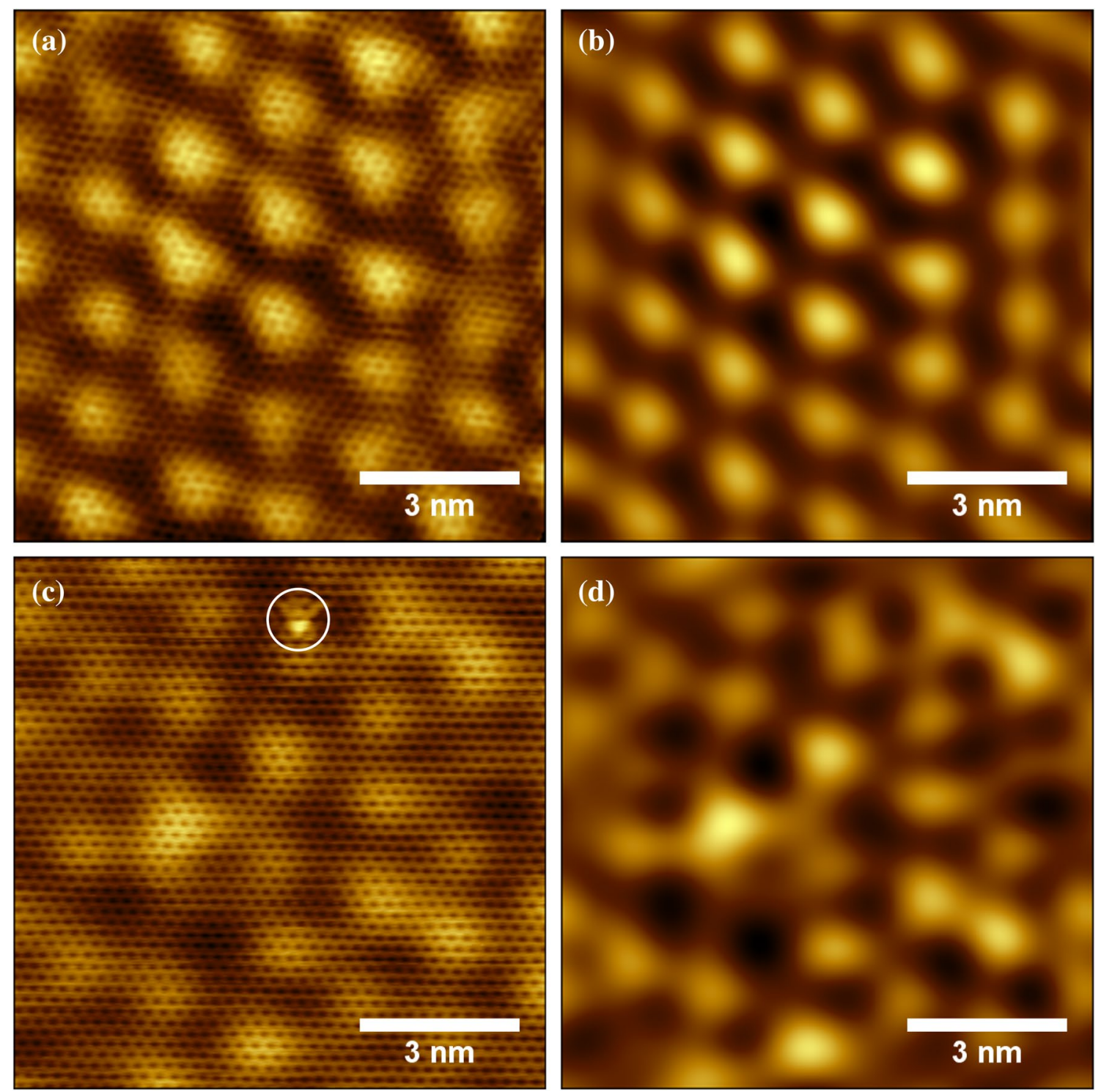

Fig. 6 Atomically resolved STM images of graphitized $\mathrm{SiC}(0001)$ samples: a, b annealed in $\mathrm{Si}$ flux of $0.8 \times 10^{14} \mathrm{~cm}^{-2} \mathrm{~s}^{-1}$; at $T=1400{ }^{\circ} \mathrm{C}$ and $t=5 \mathrm{~min}, \mathbf{c}, \mathbf{d}$ annealed in UHV at $T=1400{ }^{\circ} \mathrm{C}$ and

with earlier studies [17]. The details of the STM imaging of graphene on $\mathrm{SiC}$ (0001) have been described elsewhere [29] and the large-scale pattern has been assigned to the "quasi $6 \times 6$ corrugation", composed of the hexagons of two slightly different sizes, which combined to create the $(6 \sqrt{ } 3 \times 6 \sqrt{ } 3) \mathrm{R} 30^{\circ}$ surface lattice [30-32].

In both STM images of Fig. 6a, c, the graphene layer itself appears to be defectless regardless of the graphitization conditions. The single graphene grain is larger than the scan size and no vacancies are observed. However, for the UHV-grown sample, we observed the presence of a point defect, located most likely in the buffer layer, marked by the white circle in Fig. 6c. The presence of the defect does $t=5 \mathrm{~min}$. Maps $\mathbf{b}, \mathbf{d}$ are obtained from the maps a, $\mathbf{c}$ correspondingly, using 2D FFT filtering. Imaging parameters: a, b $I=25 \mathrm{pA}$, $U=20 \mathrm{mV} ; \mathbf{c}, \mathbf{d} I=102 \mathrm{pA}, U=20 \mathrm{mV}$

not strongly perturb the image of the local graphene lattice, making it most likely a type A defect, identified in Ref [33]. We have observed no such defects for the Si flux-assisted growth; moreover, a very pronounced difference is visible concerning the regularity of the $(6 \sqrt{3} \times 6 \sqrt{ } 3) \mathrm{R} 30^{\circ}$ subsurface lattice. We have performed $2 \mathrm{D}$ fast Fourier transform filtering to expose these patterns clearly-see Fig. $6 \mathrm{~b}$ (for the graphitization in Si flux) and Fig. 6d (for graphitization without $\mathrm{Si}$ flux). The quasi $(6 \times 6)$ pattern reminiscent of the $(6 \sqrt{3} \times 6 \sqrt{ } 3) \mathrm{R} 30^{\circ}$ surface lattice is different, depending on the preparation method. The sample annealed in UHV is characterized by a large degree of disorder-in fact, the quasi $(6 \times 6)$ pattern is barely visible. In contrast, 
graphitization in the flux of $\mathrm{Si}$ atoms results in a very regular $(6 \times 6)$ pattern. Moreover, while the regions with the disordered quasi $(6 \times 6)$ pattern are prevalent in UHV-grown samples, we found no such regions for samples annealed in Si flux. A similar type of disorder was observed in other studies, in which the method of flash annealing in UHV conditions was used to produce large-scale bilayer graphene on $6 \mathrm{H}-\mathrm{SiC}$ (0001) (for example, see Fig. 1b. in Ref. [34]). To a lesser extent, the disorder in the buffer layer is also visible for the samples prepared by atmospheric-pressure graphitization (for example, see Fig. 2a, b in Ref. [30]).

It appears that the most striking effect due to resupplying $\mathrm{Si}$ atoms to $\mathrm{SiC}$ surface during graphitization in nonequilibrium conditions is an elimination of disorder in the interfacial buffer layer. This could be very important for the electron transport in graphene on $\mathrm{SiC}$, since any irregularity in the periodic, electric potential perpendicular to the surface will contribute strongly to the scattering processes and decrease electron mobility in this material.

\section{Conclusions}

We have performed a systematic study of the graphitization of (0001) surface of silicon carbide in the flux of $\mathrm{Si}$ atoms from an external, high-purity sublimation source. Investigations of the process rate using X-ray photoelectron spectroscopy resulted in the identification of the semi-equilibrium graphitization conditions in Si flux, for which the graphene growth is characterized by a very slow rate, even as low as $0.015 \mathrm{ML} / \mathrm{min}$. The samples annealed in Si flux are characterized by a high degree of crystallographic ordering, as evidenced by STM measurements. Atomically resolved STM measurements have revealed, in particular, lower concentration of carbon nanotube or off-plane types of surface defects and large improvement in crystallographic order in the sub-surface buffer layer (or the interface reconstruction) as compared to samples annealed with no Si flux.

The graphene growth method described in this article appears to be an attractive alternative to the more popular method of graphitization of $\mathrm{SiC}$ in the presence of inert buffer gases. We assign the observed improved ordering in the graphene/ $\mathrm{SiC}(0001)$ surface system not only to the higher diffusivity of carbon atoms on the SiC surface and longer process time, but also to the very clean UHV process environment. We emphasize here that extending the graphitization time must cause increased exposition of the reacting system to interfering impurities, but this does not constitute a problem for the method presented here.

Further studies, including electrical characteristics of the obtained graphene films, are needed to describe the influence of the increased crystallographic ordering on the electrical parameters of the material. The proposed method allows to vary and freely adjust all process parameters (temperature, time and Si flux) and the absence of buffer gases makes it possible to introduce beams of other atoms acting on the surface simultaneously with silicon. This also opens up new possibilities of doping and intercalation of graphene layers already during their growth.

Acknowledgements We would like to express our gratitude to Bartosz Such for the invaluable help with the STM measurements. We would like to acknowledge the financial support of the National Science Centre, Poland (Grant no. 2014/15/N/ST5/00523) and the Polish Ministry of Science and Higher Education (Decision no. 7150/E-338/M/2015).

Open Access This article is distributed under the terms of the Creative Commons Attribution 4.0 International License (http://creativeco mmons.org/licenses/by/4.0/), which permits unrestricted use, distribution, and reproduction in any medium, provided you give appropriate credit to the original author(s) and the source, provide a link to the Creative Commons license, and indicate if changes were made.

\section{References}

1. K.S. Novoselov, V.I. Fal'Ko, L. Colombo, P.R. Gellert, M.G. Schwab, K. Kim, Nature. 490, 192-200 (2012)

2. D.V. Badami, Nature. 193, 569-570 (1962)

3. W. Norimatsu, M. Kusunokiba, Phys. Chem. Chem. Phys. 16, 3501-3511 (2014)

4. K.V. Emtsev, F. Speck, T. Seyller, L. Ley, J.D. Riley, Phys. Rev. B 77, 1-10 (2008)

5. A. Bostwick, T. Ohta, E. Microscopy, New J. Phys. 10, 023034 (2008)

6. J. Hass, R. Feng, T. Li, X. Li, Z. Zong, W.A. De Heer, P.N. First, E.H. Conrad, C.A. Jeffrey, C. Berger, Appl. Phys. Lett. 89, 143106 (2006)

7. K.V. Emtsev, A. Bostwick, K. Horn, J. Jobst, G.L. Kellogg, L. Ley, J.L. McChesney, T. Ohta, S.A. Reshanov, J. Röhrl, E. Rotenberg, A.K. Schmid, D. Waldmann, H.B. Weber, T. Seyller, Nat. Mater. 8, 203-207 (2009)

8. C. Virojanadara, M. Syväjarvi, R. Yakimova, L.I. Johansson, A.A. Zakharov, T. Balasubramanian, Phys. Rev. B 78, 245403 (2008)

9. N. Luxmi, R.M. Srivastava, Freenstra, J. Vac. Sci. Technol. B 28 C5C1 (2010)

10. N. Srivastava, G. He, P.C. Luxmi, R.M. Mende, Y. Feenstra, Sun, J. Phys. D Appl. Phys. 45, 154001 (2012)

11. R.M. Tromp, J.B. Hannon, Phys. Rev. Lett. 102, 106104 (2009)

12. W.A. de Heer, C. Berger, M. Ruan, M. Sprinkle, X. Li, Y. Hu, B. Zhang, J. Hankinson, E. Conrad, Proc. Natl. Acad. Sci. 108, 16900-16905 (2011)

13. C. Çelebi, C. Yanik, A.G. Demirkol, I.I. Kaya, Carbon 50, 30263031 (2012)

14. H.B. Jin, Y. Jeon, S. Jung, V. Modepalli, H.S. Kang, B.C. Lee, J.H. Ko, H.J. Shin, J.W. Yoo, S.Y. Kim, S.Y. Kwon, D. Eom, K. Park, Sci. Rep. 5, 1-5 (2015)

15. Q. Wang, J. Phys. Condens. Matter 25(9), 095002 (2013)

16. T.W. Hu, Nanotechnology 26, 105708 (2015)

17. J.S. Custer, M.O. Thompson, D.C. Jacobson, J.M. Poate, S. Roorda, W.C. Sinke, F. Spaepen, J.S. Custer, M. Thompson, Appl. Phys. Lett. 64(437), 437-439 (1994) 
18. Casa Software Ltd, CasaXPS: Processing Software for XPS, AES, SIMS and More, http://www.casaxps.com/. Accessed 21 June 2018

19. I.P. Wavemetrics-Overview, https://www.wavemetrics.com/ products/igorpro/igorpro.htm. Accessed 21 June 2018

20. D. Nečas, P. Klapetek, Cent. Eur. J. Phys. 10(1), 181-188 (2012)

21. J. Schindelin, I. Arganda-Carreras, E. Frise, V. Kaynig, M. Longair, T. Pietzsch, S. Preibisch, C. Rueden, S. Saalfeld, B. Schmid, J.Y. Tinevez, D.J. White, V. Hartenstein, K. Eliceiri, P. Tomancak, A. Cardona, Nat. Methods 9, 676-682 (2012)

22. D. Ferrah, J. Penuelas, C. Bottela, G. Grenet, A. Ouerghi, Surf. Sci. 615, 47-56 (2013)

23. C. Coletti, C. Riedl, D.S. Lee, B. Krauss, L. Patthey, K. Von Klitzing, J.H. Smet, U. Starke, Phys. Rev. B 81, 235401 (2010)

24. C. Xia, S. Watcharinyanon, A.A. Zakharov, R. Yakimova, L. Hultman, L.I. Johansson, C. Virojanadara, Phys. Rev. B 85, 045418 (2012)

25. C. Riedl, Epitaxial Graphene on Silicon Carbide Surfaces: Growth, Characterization, Doping and Hydrogen Intercalation, Doctoral Thesis (Friedrich-Alexander-Universität Erlangen-Nürnberg,
2010), https://opus4.kobv.de/opus4-fau/frontdoor/index/index/ docId/1364. Accessed 21 June 2018

26. H. Huang, W. Chen, S. Chen, A. Thye, S. Wee, ACS Nano 2(12), 2513-2518 (2008)

27. J.B. Hannon, R.M. Tromp, Phys. Rev. B 77, 241404 (2008)

28. N.P. Guisinger, G.M. Rutter, J.N. Crain, C. Heiliger, P.N. First, J.A. Stroscio, J. Vac. Sci. Technol. A. 26, 932-937 (2008)

29. G.M. Rutter, N.P. Guisinger, J.N. Crain, E.A.A. Jarvis, M.D. Stiles, T. Li, P.N. First, J.A. Stroscio, Phys. Rev. B 76, 235416 (2007)

30. S. Goler, C. Coletti, V. Piazza, P. Pingue, F. Colangelo, V. Pellegrini, K.V. Emtsev, S. Forti, U. Starke, F. Beltram, S. Heun, Carbon 51, 249-254 (2013)

31. U. Starke, C. Riedl, J. Phys. Condens. Matter 21, 134016 (2009)

32. W. Chen, H. Xu, L. Liu, X. Gao, D. Qi, G. Peng, S.C. Tan, Y. Feng, K.P. Loh, A.T.S. Wee, Surf. Sci. 596, 176-186 (2005)

33. G.M. Rutter, J.N. Crain, N.P. Guisinger, T. Li, P.N. First, J.A. Stroscio, Science 317(5835), 219-222 (2007)

34. Q. Wang, W. Zhang, L. Wang, K. He, X. Ma, Q. Xue, J. Phys. Condens. Matter. 25, 095002 (2013) 